\title{
Hinweise zu den Äquivalentregistern
}

Die nachfolgend abgedruckten Äquivalentregister umfassen in alphabetischer Reihenfolge sämtliche Äquivalentangaben der vier Bände. Zu den Äquivalentregistern sei auch auf das „,Vorwort der Herausgeber“ in dem ersten Band hingewiesen. Aus zuvor genannten Gründen fehlen lediglich die Äquivalentangaben zum Russischen für den Nachtrag A-H sowie für I-Z.

Die Anführung von sieben bzw. (bis zum zweiten Band) acht Äquivalentangaben zu den Lemmata des vorliegenden Wörterbuchs ist als wesentliche Bereicherung der metalexikographischen Theoriebildung zu betrachten, und hier insbesondere bei der Vermittlung der Terminologie Herbert Ernst Wiegands. Zahlreiche sprachtheoretische und weithin längst gebräuchliche metalexikographische Termini und sonstige Ausdrücke bzw. Termini werfen keine erheblichen Probleme der Übersetzung und der Rezeption auf. Was viele der komplexen Neuprägungen Herbert Ernst Wiegands angeht, so handelt es sich bei den Übersetzungen sehr oft um erste Vorschläge, denen mithin ihrerseits der Status von Neuprägungen in den hier berücksichtigten Äquivalentsprachen zukommt.

Die Äquivalentregister bieten eine ganz wesentliche Orientierung in allen schriftlichen und mündlichen Situationen, in denen man nicht hinter den erreichten Stand der Wörterbuchforschung resp. Metalexikographie zurückfallen, sondern sich an den Errungenschaften einer möglichst präzisen Bezugnahme auf lexikographische und metalexikographische Sachverhalte orientieren will, die wesentlich mit dem Namen Herbert Ernst Wiegand verbunden sind.

Nachfolgend sind die Äquivalentangaben in der gleichen Abfolge angeführt wie in der Äquivalentposition der Artikel, nämlich von ,bg“ bis „,ru“. Die automatische initialalphabetische Sortierung erfolgt durchweg ohne Berücksichtigung von Leerzeichen und Sonderzeichen (wie dem Apostroph). Für die beiden kyrillischen Alphabete des Bulgarischen und Russischen ist zu beachten, dass Namen (Zeitschriften, Jahrbücher, Institutionen) und weithin verwendete Termini des Englischen vor den Ausdrücken mit kyrillischen Buchstaben einsortiert werden. 
